Portland State University

PDXScholar

\title{
Evaluation of Route Changes Utilizing High- Resolution GPS Bus Transit Data
}

Travis Bradley Glick

Portland State University, glick.travis@gmail.com

Follow this and additional works at: https://pdxscholar.library.pdx.edu/trec_seminar

Part of the Transportation Commons, Transportation Engineering Commons, and the Urban Studies Commons

Let us know how access to this document benefits you.

\section{Recommended Citation}

Glick, Travis Bradley, "Evaluation of Route Changes Utilizing High-Resolution GPS Bus Transit Data" (2018). TREC Friday Seminar Series. 141.

https://pdxscholar.library.pdx.edu/trec_seminar/141

This Book is brought to you for free and open access. It has been accepted for inclusion in TREC Friday Seminar Series by an authorized administrator of PDXScholar. Please contact us if we can make this document more accessible: pdxscholar@pdx.edu. 


\section{Measuring the Determinants of Bus Dwell Times New Insights and Potential Data Biases \& Portland State LeAd-Author ANd PRESENTER TRAVIS B. GLICK




\section{Who am I?}

- Travis Bradley Glick

- Portland State University

- Transportation, Technology, and People Lab

- Transportation Research And Innovation Lab

-Advisor: Miguel A. Figliozzi

- Undergraduate Research and Thesis

- Ongoing Research (Today's Subject)

- Master's Thesis

- Doctoral Research

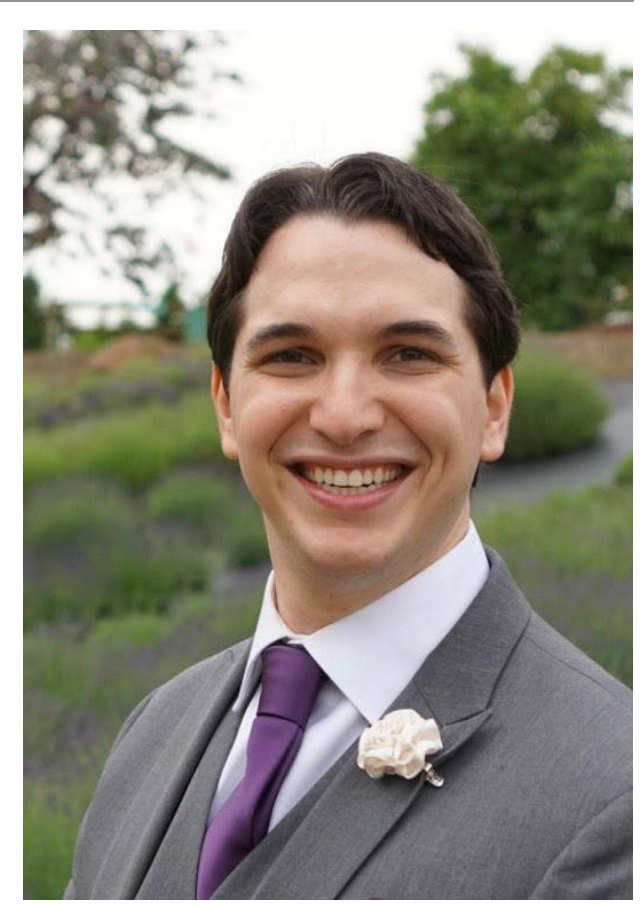

- 5 peer-reviewed publications

- 2015, 2016, (2) 2017, and 2018 Transportation Research Record 


\section{Project Motivation}

- Dwell time contributes to travel-time variability

- Travel-time variability affects operator costs and user satisfaction

- Previous researched has studied key factors of dwell time variability

- Relied on stop-level data

- Low reliability for stops near signalized intersections

- Biases when estimating coefficients of passenger movements

- New data sources provide new avenues of research

- New variables

- Bias Corrections? 


\section{Project Overview}

- Individual Stops

- Midblock Stops

- Nearside Stops

- Farside Stops

- Timepoint Stops

- Pooled Results from all stop types

- New Independent and Dependent Variables 


\section{Data Sources - Stop Level}

- Stop Level

- Data collected at the bus stops

- Ons, Offs, Lift, Dwell, Door Operations, Door Open Time

- Widespread implementation

-Widely used to analyze routes, segments, and bus stops

- Well-studied 


\section{Study Area}

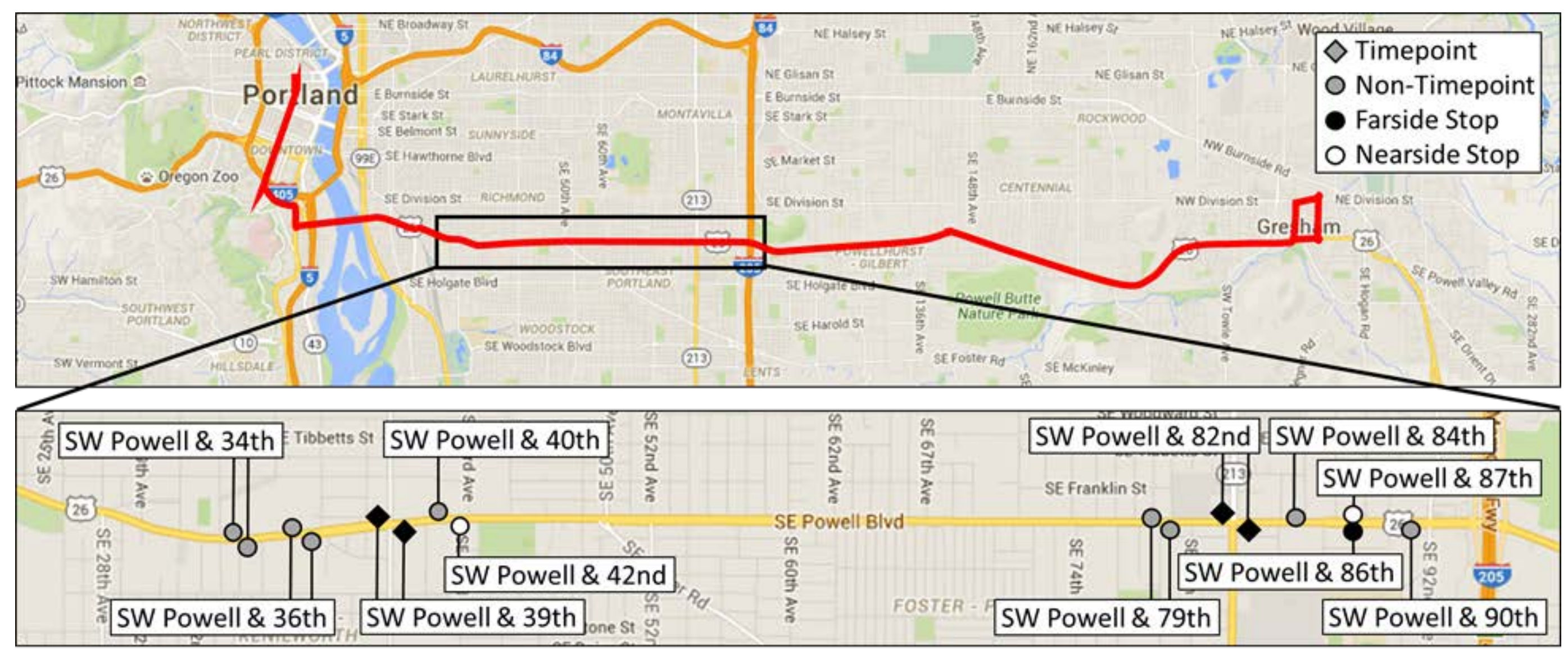




\section{High-Resolution Data}

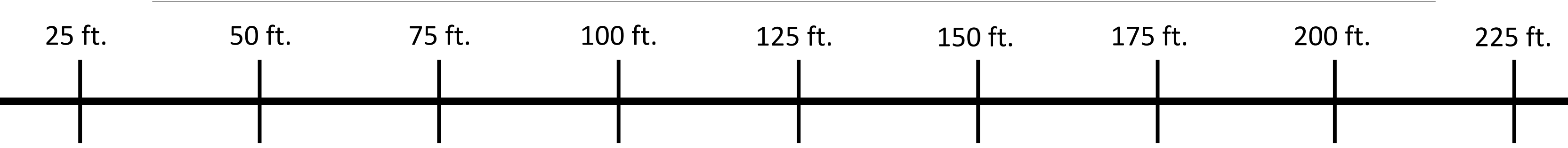

Time 1

Time 2

Time 3 


\section{Data Sources - Combined Data}

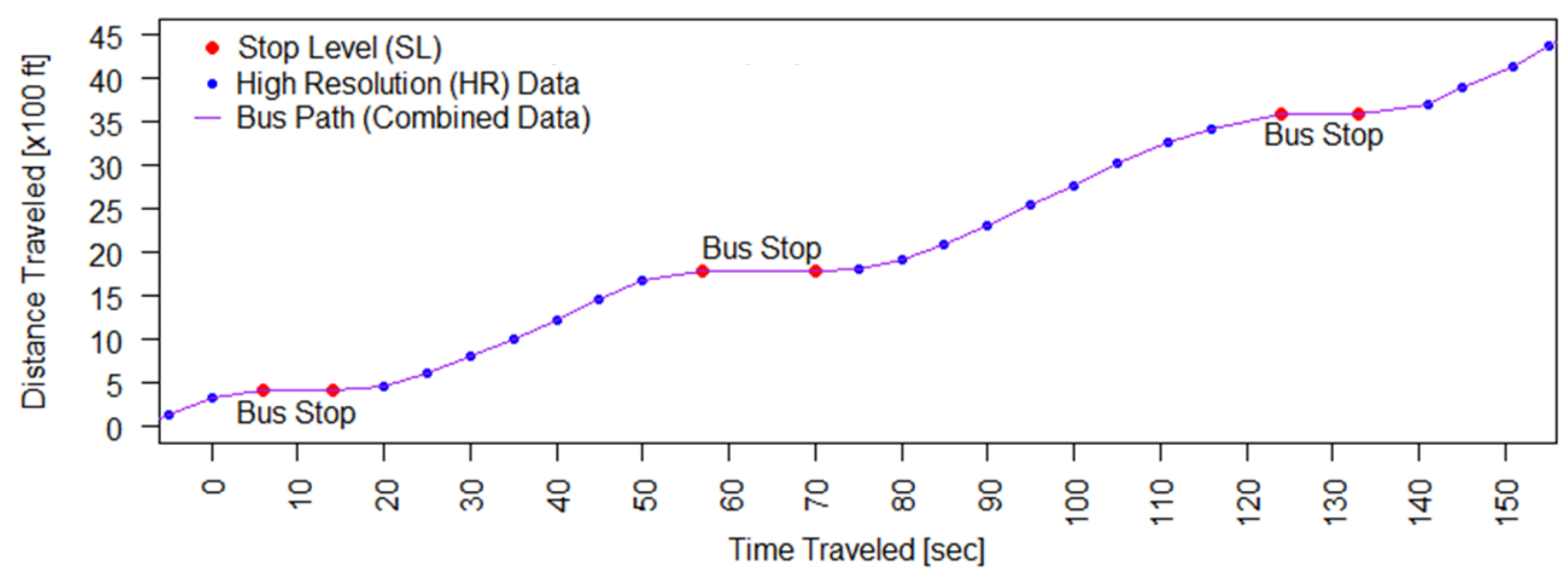




\section{Dwell Statistics}

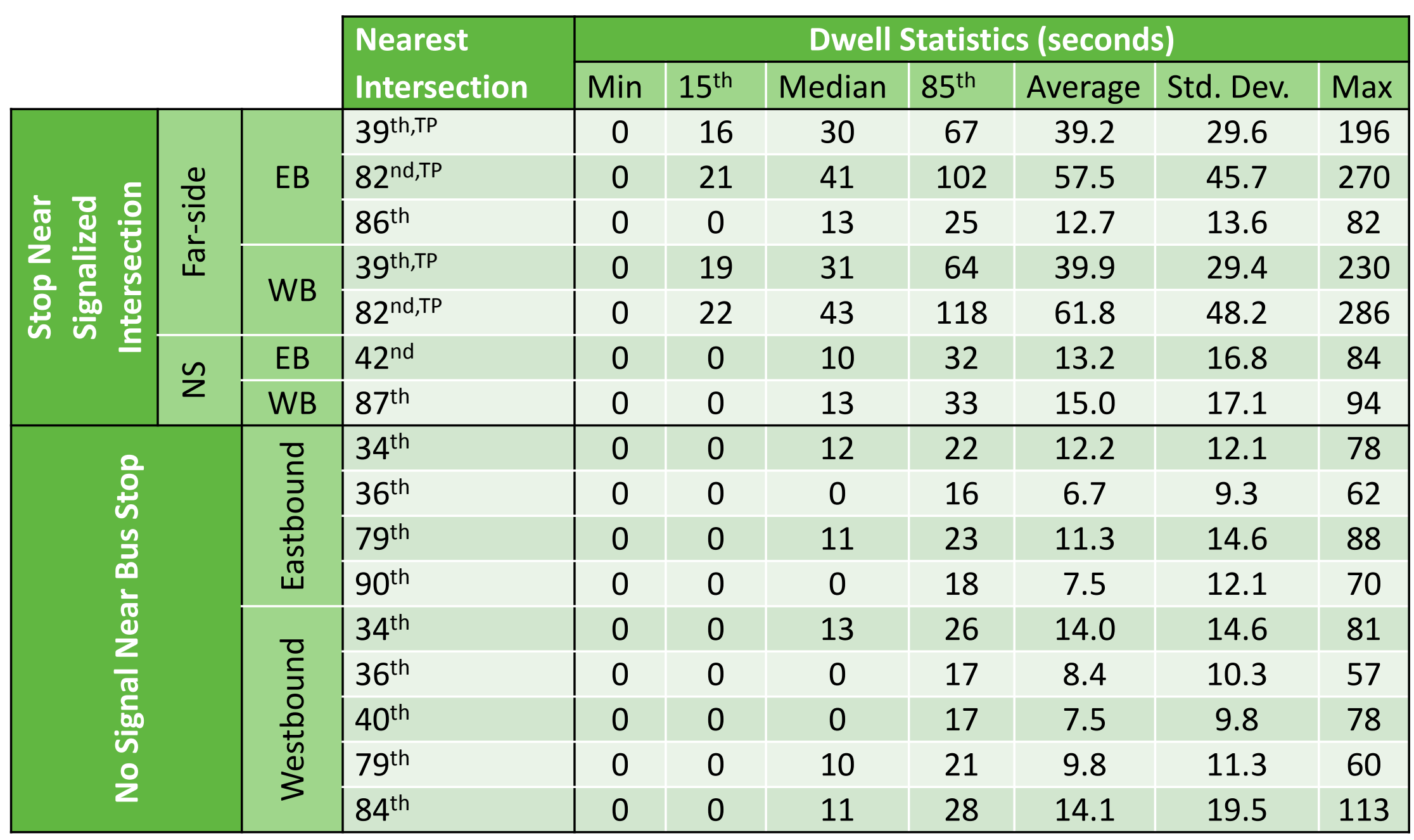




\section{Dwell Statistics}

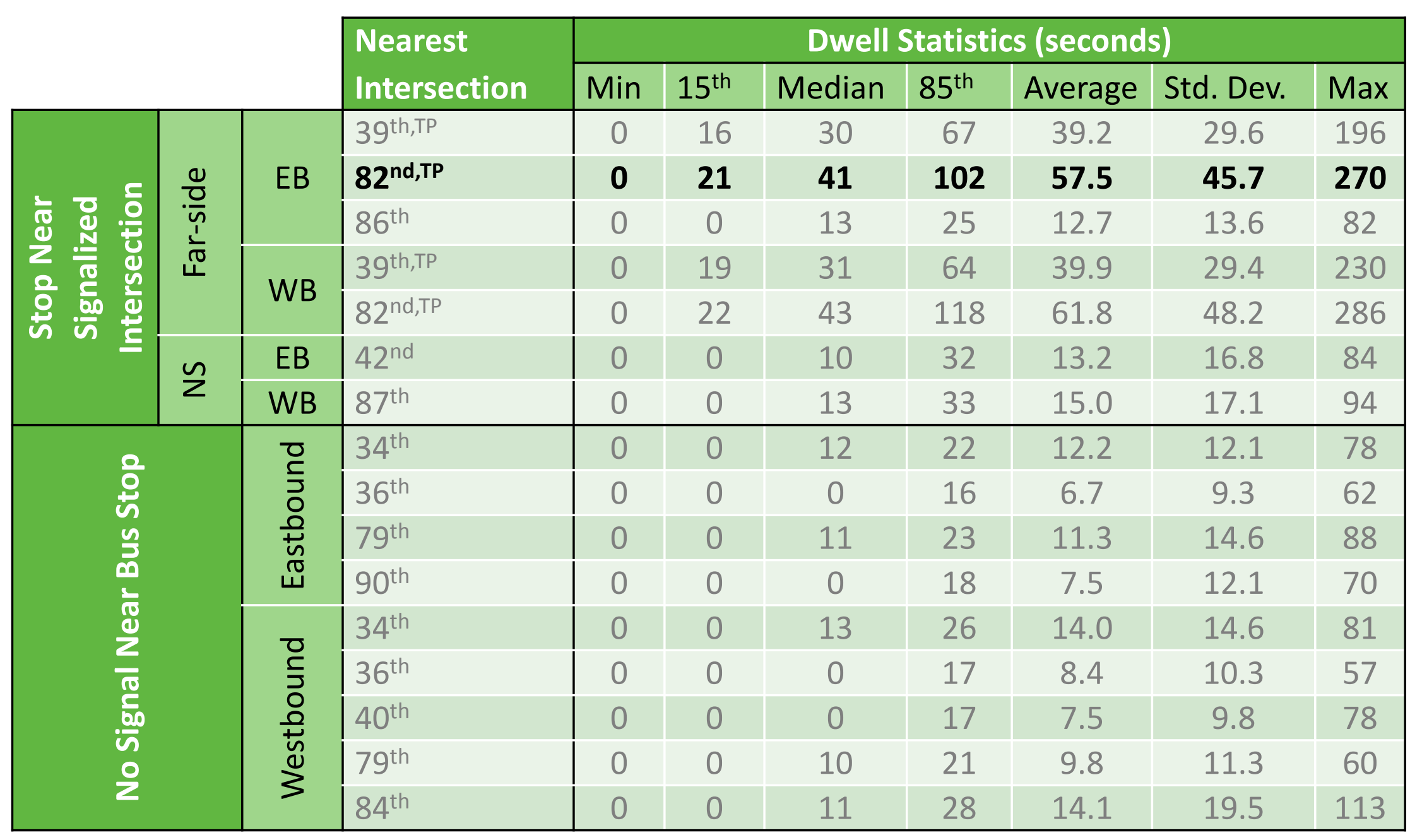




\section{Dwell Statistics}

\begin{tabular}{|c|c|c|c|c|c|c|c|c|c|c|}
\hline & \multirow{2}{*}{$\begin{array}{l}\text { Nearest } \\
\text { Intersection }\end{array}$} & \multicolumn{7}{|c|}{ Dwell Statistics (seconds) } \\
\hline & & & & Min & $15^{\text {th }}$ & Median & $85^{\text {th }}$ & Average & Std. Dev. & Max \\
\hline \multirow{7}{*}{ 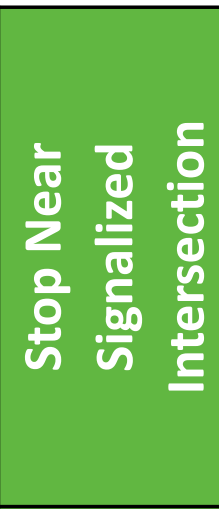 } & \multirow{5}{*}{$\begin{array}{l}\frac{0}{0} \\
\frac{1}{0} \\
\frac{1}{4}\end{array}$} & \multirow{3}{*}{ EB } & $39^{\mathrm{th}, \mathrm{TP}}$ & 0 & 16 & 30 & 67 & 39.2 & 29.6 & 196 \\
\hline & & & $82^{\text {nd,TP }}$ & 0 & 21 & 41 & 102 & 57.5 & 45.7 & 270 \\
\hline & & & $86^{\text {th }}$ & 0 & 0 & 13 & 25 & 12.7 & 13.6 & 82 \\
\hline & & \multirow{2}{*}{ WB } & $39^{\text {th, TP }}$ & 0 & 19 & 31 & 64 & 39.9 & 29.4 & 230 \\
\hline & & & $82^{\text {nd,TP }}$ & 0 & 22 & 43 & 118 & 61.8 & 48.2 & 286 \\
\hline & \multirow{2}{*}{$\Sigma$} & $E B$ & $42^{\text {nd }}$ & 0 & 0 & 10 & 32 & 13.2 & 16.8 & 84 \\
\hline & & WB & $87^{\text {th }}$ & 0 & 0 & 13 & 33 & 15.0 & 17.1 & 94 \\
\hline \multirow{9}{*}{\multicolumn{2}{|c|}{ 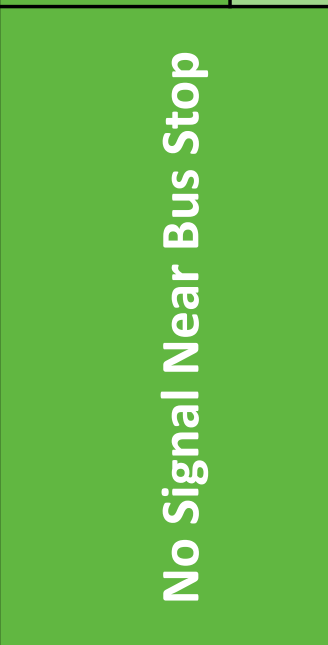 }} & \multirow{4}{*}{ 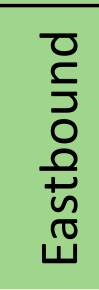 } & $34^{\text {th }}$ & 0 & 0 & 12 & 22 & 12.2 & 12.1 & 78 \\
\hline & & & $36^{\text {th }}$ & 0 & 0 & 0 & 16 & 6.7 & 9.3 & 62 \\
\hline & & & $79^{\text {th }}$ & 0 & 0 & 11 & 23 & 11.3 & 14.6 & 88 \\
\hline & & & $90^{\text {th }}$ & 0 & 0 & 0 & 18 & 7.5 & 12.1 & 70 \\
\hline & & \multirow{5}{*}{ 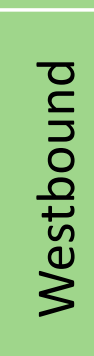 } & $34^{\text {th }}$ & 0 & 0 & 13 & 26 & 14.0 & 14.6 & 81 \\
\hline & & & $36^{\text {th }}$ & 0 & 0 & 0 & 17 & 8.4 & 10.3 & 57 \\
\hline & & & $40^{\text {th }}$ & 0 & 0 & 0 & 17 & 7.5 & 9.8 & 78 \\
\hline & & & $79^{\text {th }}$ & 0 & 0 & 10 & 21 & 9.8 & 11.3 & 60 \\
\hline & & & $84^{\text {th }}$ & 0 & 0 & 11 & 28 & 14.1 & 19.5 & 113 \\
\hline
\end{tabular}




\section{Stop Activity}

\begin{tabular}{|c|c|c|c|c|c|c|c|c|c|c|}
\hline & & & & $\begin{array}{l}\text { Nearest } \\
\text { Intersection }\end{array}$ & $\begin{array}{c}\text { Stops } \\
\text { (per day) }\end{array}$ & $\begin{array}{c}\text { Ons } \\
\text { (per day) }\end{array}$ & $\begin{array}{c}\text { Offis } \\
\text { (per day) }\end{array}$ & $\begin{array}{c}\text { Lift }^{*} \\
\text { (per week) } \\
\end{array}$ & $\begin{array}{l}\text { Bus } \\
\text { Bay } \\
\end{array}$ & Shelter \\
\hline \multirow{7}{*}{ 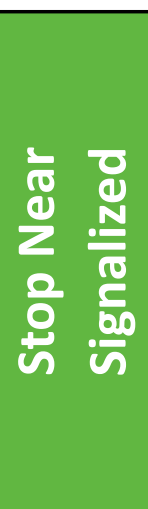 } & \multirow{7}{*}{ 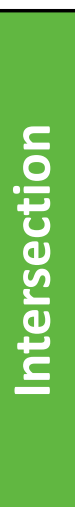 } & \multirow{5}{*}{$\begin{array}{l}\frac{0}{0} \\
\frac{1}{5} \\
\frac{1}{1} \\
\amalg\end{array}$} & \multirow{3}{*}{$\mathrm{EB}$} & $39^{\text {th,TP }}$ & 63 & 162 & 283 & 9.0 & $x$ & $x$ \\
\hline & & & & $82^{\text {nd,TP }}$ & 64 & 256 & 266 & 15.0 & $x$ & $x$ \\
\hline & & & & $86^{\text {th }}$ & 40 & 32 & 55 & 1.0 & $x$ & $x$ \\
\hline & & & \multirow{2}{*}{ WB } & $39^{\text {th,TP }}$ & 64 & 268 & 110 & 6.0 & & $x$ \\
\hline & & & & $82^{\mathrm{nd}, \mathrm{TP}}$ & 63 & 226 & 155 & 5.3 & $x$ & $x$ \\
\hline & & \multirow{2}{*}{ 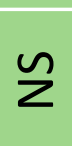 } & $\mathrm{EB}$ & $42^{\text {nd }}$ & 33 & 12 & 40 & 0.3 & & $x$ \\
\hline & & & WB & $87^{\text {th }}$ & 38 & 48 & 30 & 1.0 & $x$ & \\
\hline \multirow{9}{*}{\multicolumn{2}{|c|}{ 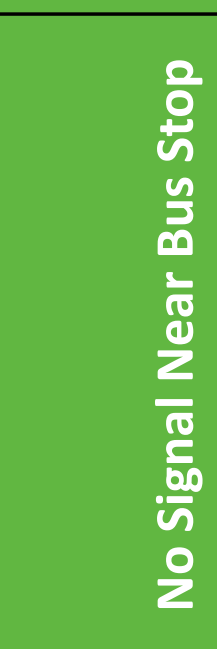 }} & & \multirow{4}{*}{ 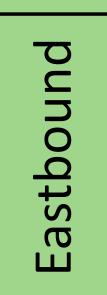 } & $34^{\text {th }}$ & 45 & 35 & 70 & 2.0 & & \\
\hline & & & & $36^{\text {th }}$ & 29 & 11 & 35 & 0.3 & & \\
\hline & & & & $79^{\text {th }}$ & 35 & 8 & 63 & 1.0 & $x$ & \\
\hline & & & & $90^{\text {th }}$ & 22 & 10 & 26 & 0.3 & & $x$ \\
\hline & & & \multirow{5}{*}{ 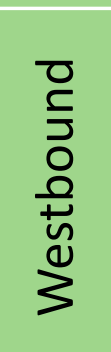 } & $34^{\text {th }}$ & 45 & 76 & 32 & 2.7 & & $x$ \\
\hline & & & & $36^{\text {th }}$ & 32 & 41 & 16 & 0.3 & & \\
\hline & & & & $40^{\text {th }}$ & 31 & 15 & 42 & 1.3 & & \\
\hline & & & & $79^{\text {th }}$ & 35 & 54 & 7 & 0.0 & $x$ & $x$ \\
\hline & & & & $84^{\text {th }}$ & 34 & 16 & 71 & 2.3 & $x$ & \\
\hline
\end{tabular}




\section{Stop Activity}

\begin{tabular}{|c|c|c|c|c|c|c|c|c|c|c|}
\hline & & & & $\begin{array}{l}\text { Nearest } \\
\text { Intersection }\end{array}$ & $\begin{array}{c}\text { Stops } \\
\text { (per day) }\end{array}$ & $\begin{array}{c}\text { Ons } \\
\text { (per day) }\end{array}$ & $\begin{array}{c}\text { Offis } \\
\text { (per day) }\end{array}$ & $\begin{array}{c}\text { Lift }^{*} \\
\text { (per week) } \\
\end{array}$ & $\begin{array}{l}\text { Bus } \\
\text { Bay } \\
\end{array}$ & Shelter \\
\hline \multirow{7}{*}{ 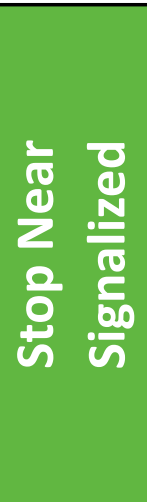 } & \multirow{7}{*}{ 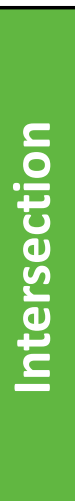 } & \multirow{5}{*}{$\begin{array}{l}\frac{0}{0} \\
\frac{0}{n} \\
\frac{1}{\pi} \\
\frac{1}{4}\end{array}$} & \multirow{3}{*}{$\mathrm{EB}$} & $39^{\text {th,TP }}$ & 63 & 162 & 283 & 9.0 & $x$ & $x$ \\
\hline & & & & $82^{n d, T P}$ & 64 & 256 & 266 & 15.0 & $x$ & $x$ \\
\hline & & & & $86^{\text {th }}$ & 40 & 32 & 55 & 1.0 & $x$ & $x$ \\
\hline & & & \multirow{2}{*}{ WB } & $39^{\text {th,TP }}$ & 64 & 268 & 110 & 6.0 & & $x$ \\
\hline & & & & $82^{\text {nd,TP }}$ & 63 & 226 & 155 & 5.3 & $x$ & $x$ \\
\hline & & \multirow{2}{*}{ 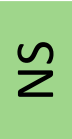 } & $\mathrm{EB}$ & $42^{\text {nd }}$ & 33 & 12 & 40 & 0.3 & & $x$ \\
\hline & & & WB & $87^{\text {th }}$ & 38 & 48 & 30 & 1.0 & $x$ & \\
\hline \multirow{9}{*}{\multicolumn{2}{|c|}{ 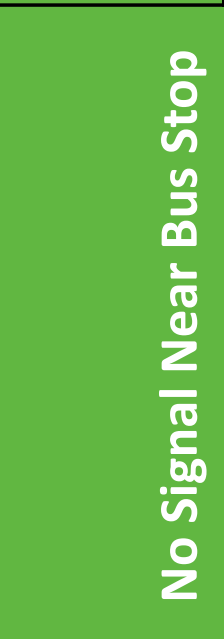 }} & & \multirow{4}{*}{ 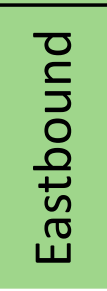 } & $34^{\text {th }}$ & 45 & 35 & 70 & 2.0 & & \\
\hline & & & & $36^{\text {th }}$ & 29 & 11 & 35 & 0.3 & & \\
\hline & & & & $79^{\text {th }}$ & 35 & 8 & 63 & 1.0 & $x$ & \\
\hline & & & & $90^{\text {th }}$ & 22 & 10 & 26 & 0.3 & & $x$ \\
\hline & & & \multirow{5}{*}{ 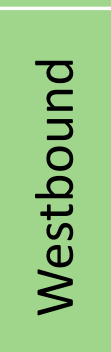 } & $34^{\text {th }}$ & 45 & 76 & 32 & 2.7 & & $x$ \\
\hline & & & & $36^{\text {th }}$ & 32 & 41 & 16 & 0.3 & & \\
\hline & & & & $40^{\text {th }}$ & 31 & 15 & 42 & 1.3 & & \\
\hline & & & & $79^{\text {th }}$ & 35 & 54 & 7 & 0.0 & $x$ & $x$ \\
\hline & & & & $84^{\text {th }}$ & 34 & 16 & 71 & 2.3 & $x$ & \\
\hline
\end{tabular}




\section{Stop Activity}

\begin{tabular}{|c|c|c|c|c|c|c|c|c|c|c|}
\hline & & & & $\begin{array}{l}\text { Nearest } \\
\text { Intersection }\end{array}$ & $\begin{array}{c}\text { Stops } \\
\text { (per day) }\end{array}$ & $\begin{array}{c}\text { Ons } \\
\text { (per day) }\end{array}$ & $\begin{array}{c}\text { Offis } \\
\text { (per day) }\end{array}$ & $\begin{array}{c}\text { Lift }^{*} \\
\text { (per week) } \\
\end{array}$ & $\begin{array}{l}\text { Bus } \\
\text { Bay } \\
\end{array}$ & Shelter \\
\hline \multirow{7}{*}{ 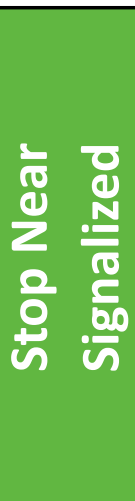 } & \multirow{7}{*}{ 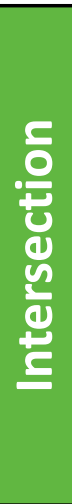 } & \multirow{5}{*}{$\begin{array}{l}\frac{0}{0} \\
\frac{0}{n} \\
\frac{1}{\pi} \\
\frac{1}{4}\end{array}$} & \multirow{3}{*}{$\mathrm{EB}$} & $39^{\text {th,TP }}$ & 63 & 162 & 283 & 9.0 & $x$ & $x$ \\
\hline & & & & $82^{\text {nd,TP }}$ & 64 & 256 & 266 & 15.0 & $x$ & $x$ \\
\hline & & & & $86^{\text {th }}$ & 40 & 32 & 55 & 1.0 & $x$ & $x$ \\
\hline & & & \multirow{2}{*}{ WB } & $39^{\text {th,TP }}$ & 64 & 268 & 110 & 6.0 & & $x$ \\
\hline & & & & $82^{\text {nd,TP }}$ & 63 & 226 & 155 & 5.3 & $x$ & $x$ \\
\hline & & \multirow{2}{*}{ 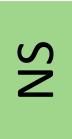 } & $\mathrm{EB}$ & $42^{\text {nd }}$ & 33 & 12 & 40 & 0.3 & & $x$ \\
\hline & & & WB & $87^{\text {th }}$ & 38 & 48 & 30 & 1.0 & $x$ & \\
\hline \multirow{9}{*}{\multicolumn{2}{|c|}{$\begin{array}{l}\frac{0}{0} \\
\frac{3}{n} \\
\frac{4}{2} \\
0 \\
\frac{2}{0} \\
\frac{1}{2} \\
\frac{\pi}{0} \\
\frac{0}{00} \\
\frac{0}{2} \\
\frac{0}{2}\end{array}$}} & & \multirow{4}{*}{ 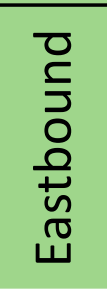 } & $34^{\text {th }}$ & 45 & 35 & 70 & 2.0 & & \\
\hline & & & & $36^{\text {th }}$ & 29 & 11 & 35 & 0.3 & & \\
\hline & & & & $79^{\text {th }}$ & 35 & 8 & 63 & 1.0 & $x$ & \\
\hline & & & & $90^{\text {th }}$ & 22 & 10 & 26 & 0.3 & & $x$ \\
\hline & & & \multirow{5}{*}{ 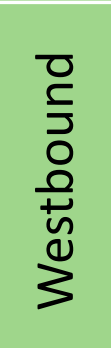 } & $34^{\text {th }}$ & 45 & 76 & 32 & 2.7 & & $x$ \\
\hline & & & & $36^{\text {th }}$ & 32 & 41 & 16 & 0.3 & & \\
\hline & & & & $40^{\text {th }}$ & 31 & 15 & 42 & 1.3 & & \\
\hline & & & & $79^{\text {th }}$ & 35 & 54 & 7 & 0.0 & $x$ & $x$ \\
\hline & & & & $84^{\text {th }}$ & 34 & 16 & 71 & 2.3 & $x$ & \\
\hline
\end{tabular}




\section{Variables (Stop Level Data)}

- Ons: number of passengers boarding a bus at a specific stop (passengers board only from front door)

- Offs: number of passengers alighting a bus at a specific stop (passengers alight from both doors)

- Lift: number that indicates wheelchair lift operation

- Early: number of minutes ahead of schedule at a time-point

- Stop: binary variable equal to one if a bus stopped to serve passengers and equal to zero otherwise 


\section{Variables (Combined Data)}

- Length: distance in-between consecutive GPS coordinates before and after the bus stop

- Speed: average speed of bus in the segment immediately before and immediately after the bus bay

- Nearside: Binary variable indicating if a stop is located on the nearside of a signalized intersection

- Farside: Binary variable indicating if a stop is located on the farside of a signalized intersection 


\section{Nearside Stop}

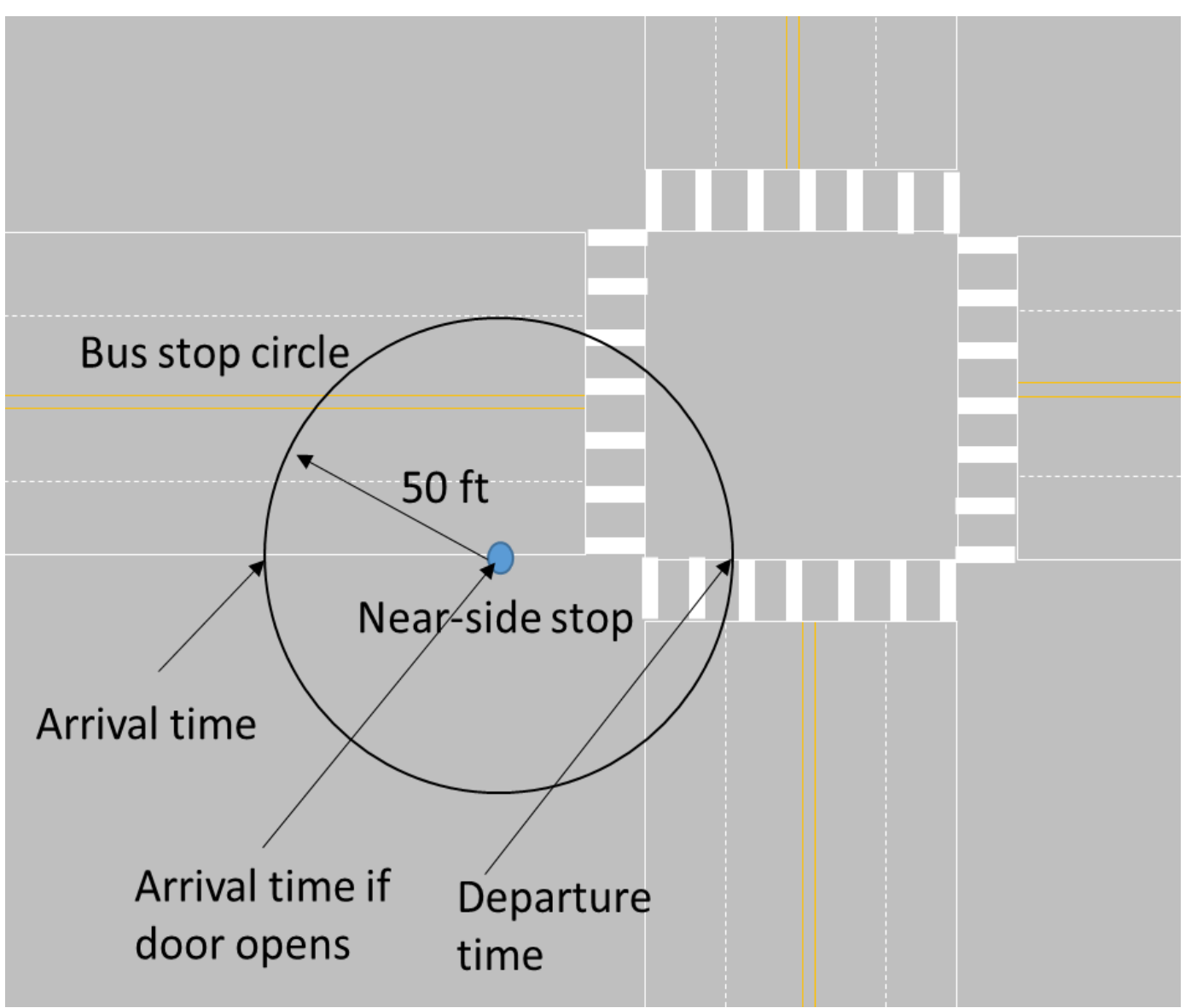




\section{Farside Stop}

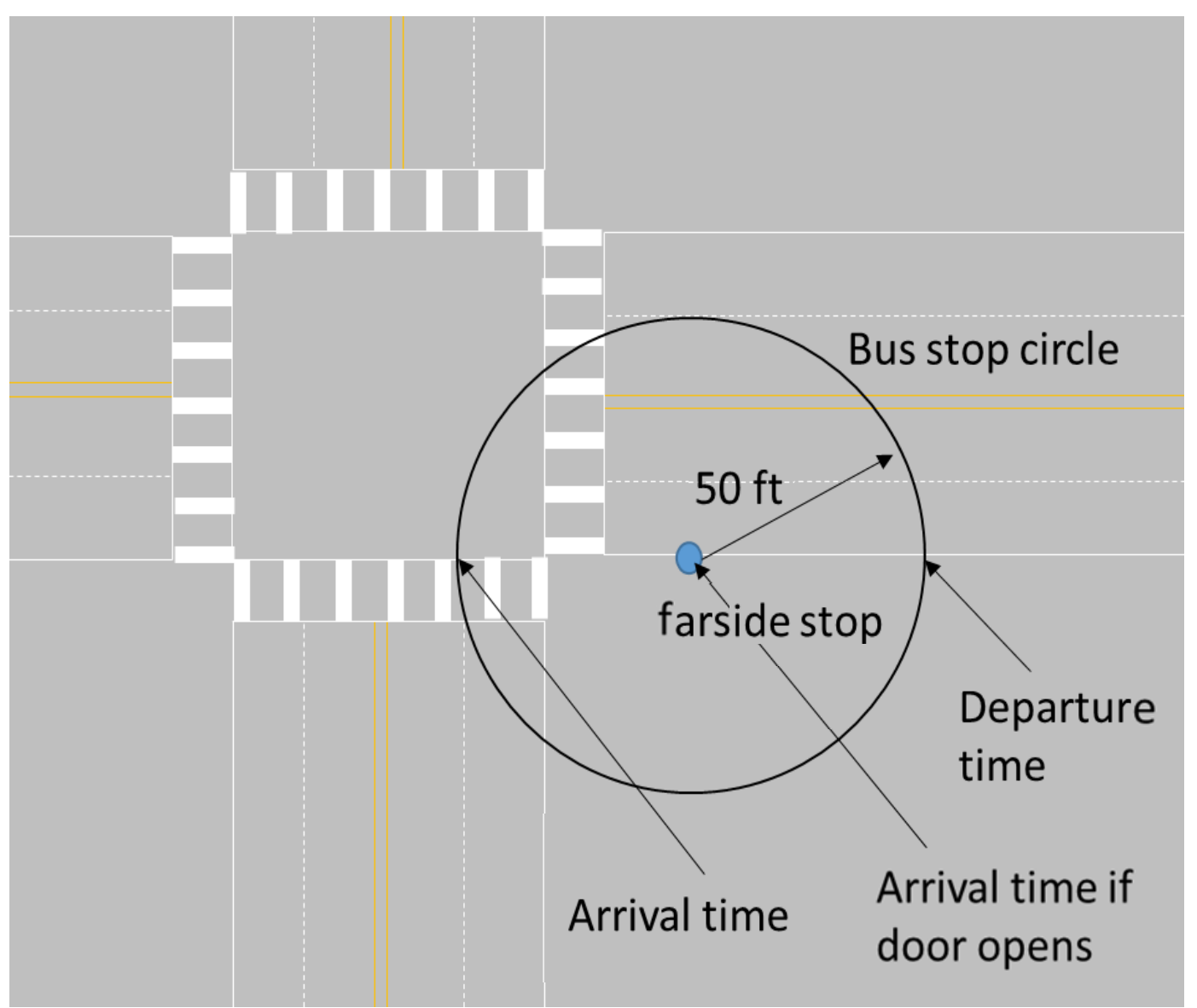




\section{Variables (Combined Data)}

- Intersection: binary variable, equal to one if a GPS point is located on the other side of a signalized intersection with respect to a bus stop. This variable has non-zero values only for near-side or far-side stops

- Int-far: binary variable, equal to one if a GPS point is located on the nearside of a farside bus stop

- Int-near: binary variable, equal to one if a GPS point is located on the farside of a nearside bus stop 


\section{Intersection}

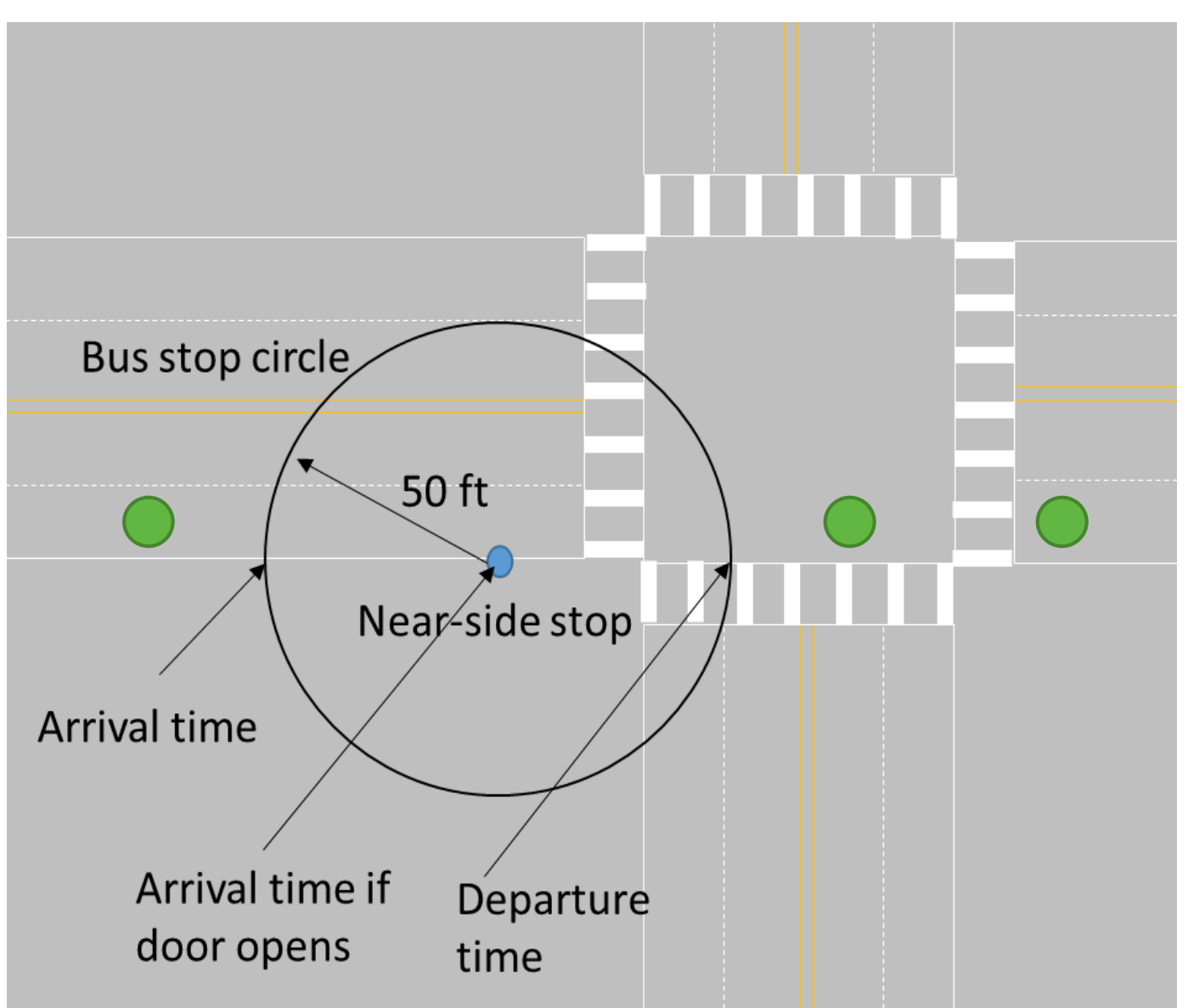




\section{Models}

- A, B - Dwell Time from the stop-level data as the dependent variables - Only passenger activity variables are included.

- C, D - Dwell time from the stop-level data as the dependent variable - All available dependent variables are included.

- E, F - Interval time from the combined data as the dependent variable - All available dependent variables are included.

- A, B, C (no lift movements) 


\begin{tabular}{|c|c|c|c|c|c|}
\hline \multicolumn{2}{|c|}{$R_{a d j}^{2}$} & Bus Stop Location & $\begin{array}{l}\text { (B) Dwell Stops + } \\
\text { Ons + Offs + Lift }\end{array}$ & $\begin{array}{l}\text { (D) Dwell Stops + Ons + } \\
\text { Offs + Lift + Avg. Speed + } \\
\text { Early + Intersection }\end{array}$ & $\begin{array}{l}\text { (F) Interval Stops + } \\
\text { Ons + Offs + Lift + } \\
\text { Avg. Speed + Length + } \\
\text { Early + Intersection }\end{array}$ \\
\hline \multirow{4}{*}{ ఏ } & \multirow{2}{*}{ EB } & Powell \& $3^{\text {th }, \mathrm{FS}}$ & 0.165 & 0.499 & 0.600 \\
\hline & & Powell $\& 82^{\text {nd, Fs }}$ & 0.135 & 0.414 & 0.574 \\
\hline & \multirow{2}{*}{ WB } & Powell \& 39th,Fs & 0.189 & 0.431 & 0.476 \\
\hline & & Powell $\& 82^{\text {nd,Fs }}$ & 0.122 & 0.250 & 0.471 \\
\hline \multirow{12}{*}{ 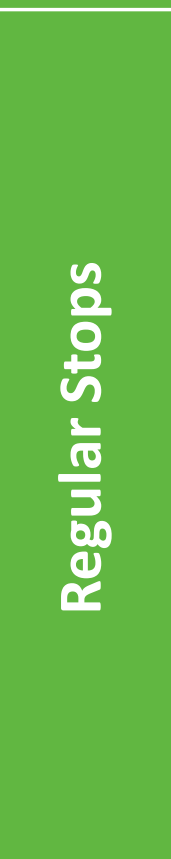 } & \multirow{6}{*}{ 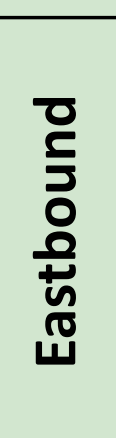 } & Powell \& 34th & 0.670 & 0.671 & 0.666 \\
\hline & & Powell \& $36^{\text {th }}$ & 0.787 & 0.787 & 0.578 \\
\hline & & Powell $\& 42^{\text {nd,NS }}$ & 0.553 & 0.553 & 0.522 \\
\hline & & Powell \& $7^{\text {th }}$ & 0.611 & 0.623 & 0.587 \\
\hline & & Powell $\& 86^{\text {th,Fs }}$ & 0.737 & 0.737 & 0.737 \\
\hline & & Powell $\& 90^{\text {th }}$ & 0.731 & 0.733 & 0.699 \\
\hline & \multirow{6}{*}{ 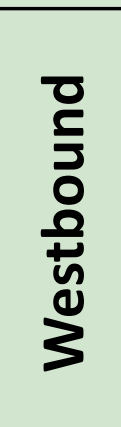 } & Powell \& $34^{\text {th }}$ & 0.719 & 0.719 & 0.693 \\
\hline & & Powell \& $36^{\text {th }}$ & 0.847 & 0.848 & 0.729 \\
\hline & & Powell \& $40^{\text {th }}$ & 0.811 & 0.814 & 0.842 \\
\hline & & Powell \& 79th & 0.795 & 0.795 & 0.788 \\
\hline & & Powell $\& 84^{\text {th }}$ & 0.554 & 0.561 & 0.533 \\
\hline & & Powell $\& 87^{\text {th,Ns }}$ & 0.687 & 0.701 & 0.684 \\
\hline
\end{tabular}

NS = near-side bus stop $\quad F S=$ far-side bus stop 


\section{$R_{a d j}^{2}$}

Ren
(B) Dwell Stops +

Ons + Offs + Lift
(D) Dwell Stops + Ons + Early + Intersection Offs + Lift + Avg. Speed +

(F) Interval Stops + Ons + Offs + Lift + Avg. Speed + Length + Early + Intersection

$\mathbf{0 . 4 9 9}$

0.414

\begin{tabular}{|l|l|}
\hline 0.431 & 0.476 \\
\hline 0.250 & 0.471 \\
\hline 0.671 & 0.666 \\
\hline 0.787 & 0.578 \\
\hline 0.553 & 0.522 \\
\hline 0.623 & 0.587 \\
\hline 0.737 & 0.737 \\
\hline 0.733 & 0.699 \\
\hline 0.719 & 0.693 \\
\hline 0.848 & 0.729 \\
\hline 0.814 & 0.842 \\
\hline 0.795 & 0.788 \\
\hline 0.561 & 0.533 \\
\hline 0.701 & 0.684 \\
\hline
\end{tabular}

NS = near-side bus stop $\quad F S=$ far-side bus stop

$\begin{array}{r}\mathbf{0 . 1 6 5} \\ 0.135 \\ \hline 0.189 \\ 0.122 \\ 0.670 \\ 0.787 \\ 0.553 \\ 0.611 \\ 0.737 \\ 0.731 \\ 0.719 \\ 0.847 \\ 0.811 \\ 0.795 \\ 0.554 \\ 0.687 \\ \hline \text { stop }\end{array}$




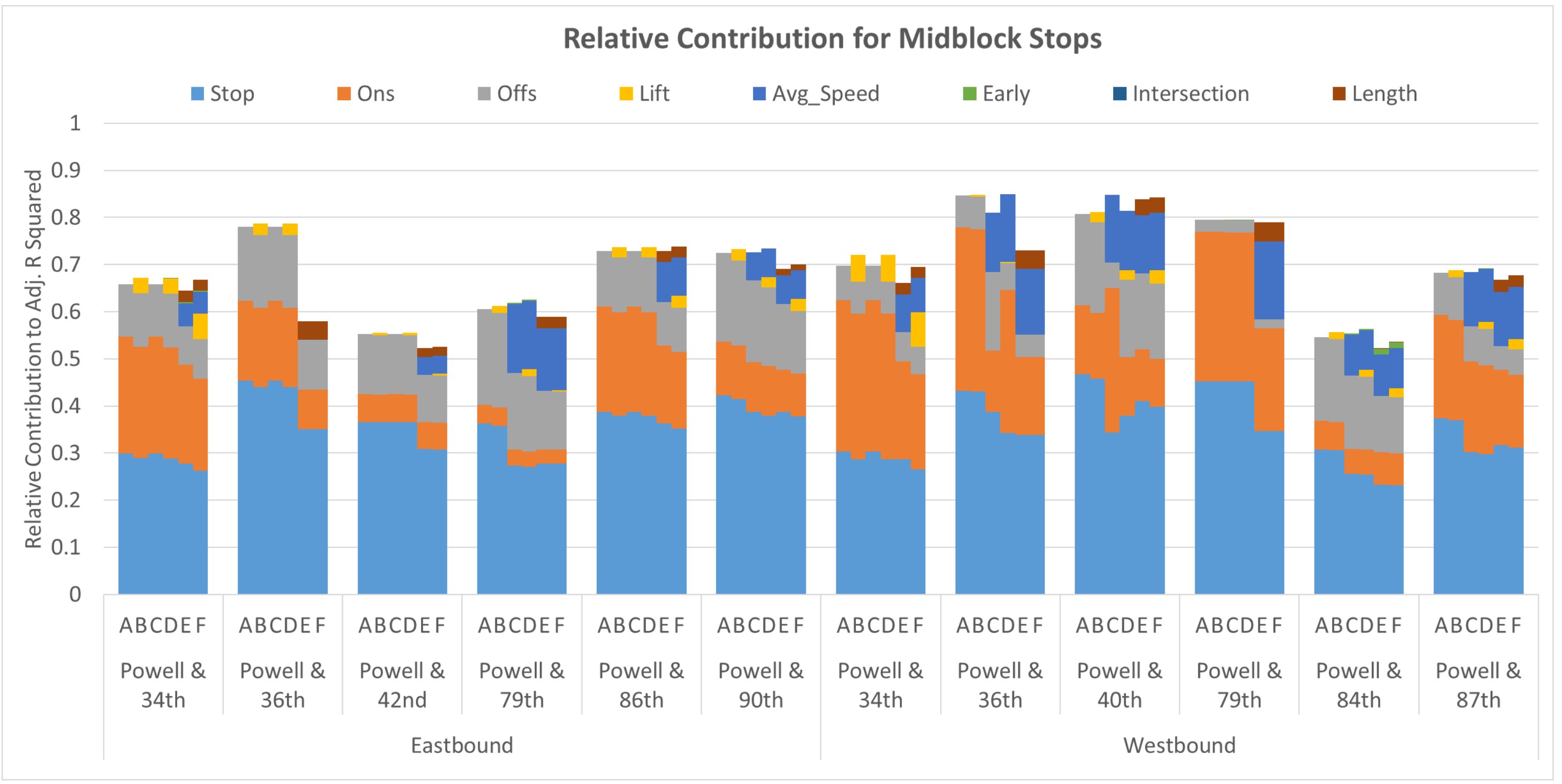




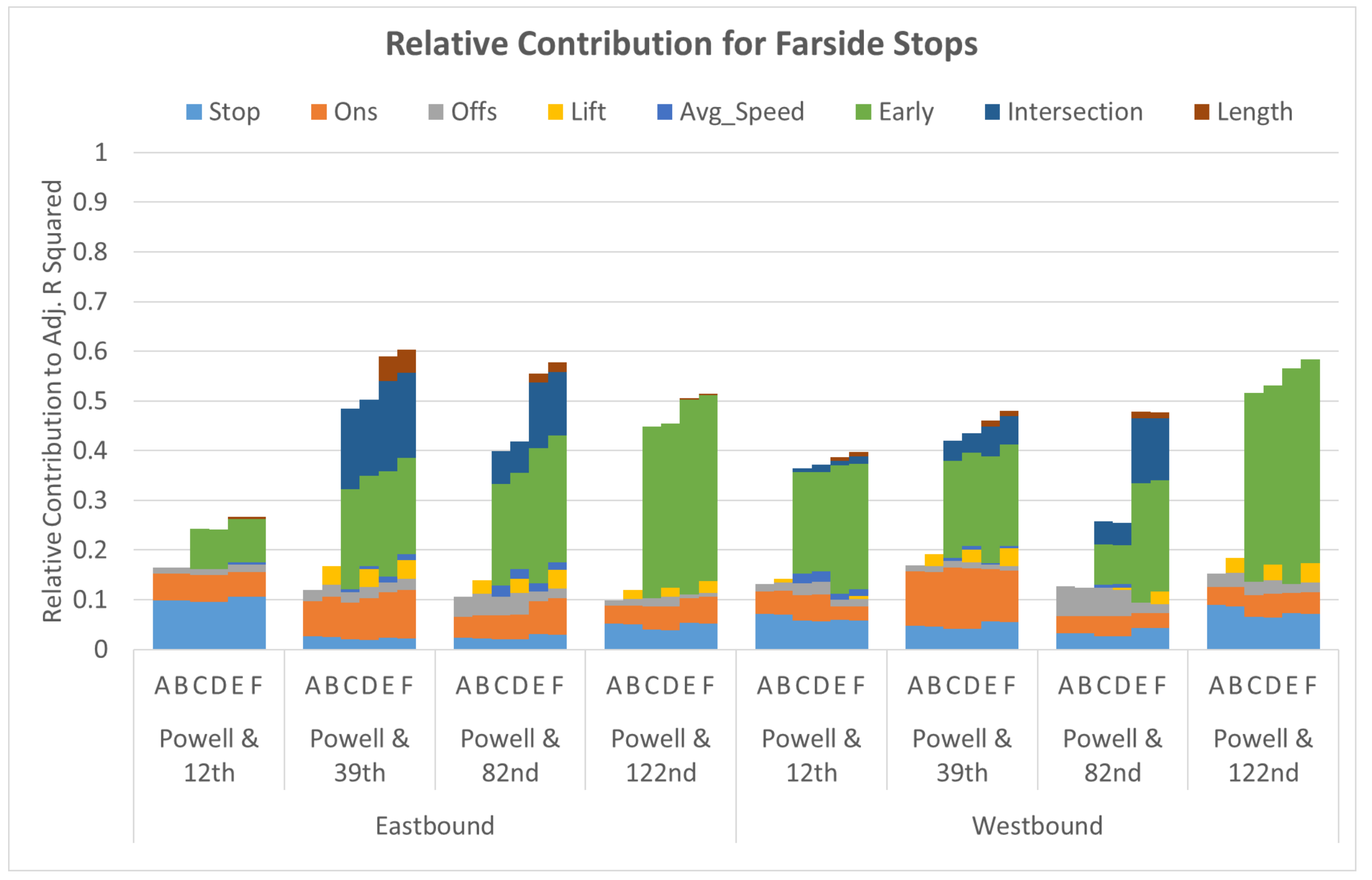




\section{$82^{\text {nd }}$ Model F}

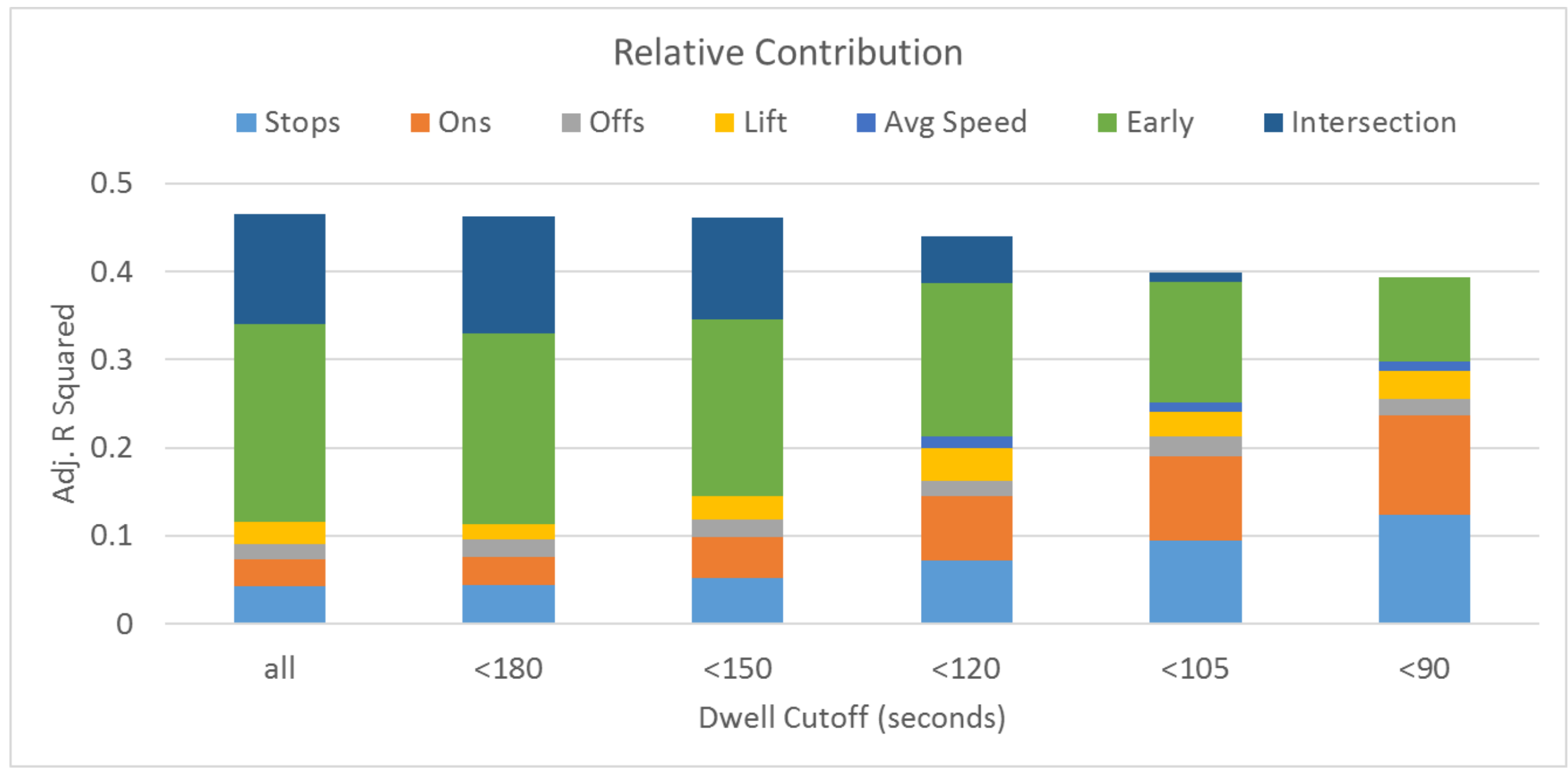


Pooled Results

\begin{tabular}{|c|c|c|c|c|}
\hline & \multicolumn{4}{|c|}{ Model (F) - Interval } \\
\hline & Coefficient & $\begin{array}{l}\text { Relative } \\
\text { Contribution }\end{array}$ & Std. Error & $\begin{array}{l}\text { Variance } \\
\text { Inflation } \\
\text { Factor }\end{array}$ \\
\hline Intercept & -0.501 & & 0.788 & \\
\hline Stops & $16.43^{* * *}$ & $13.04 \%$ & 0.392 & 1.450 \\
\hline Ons & $4.041^{* * *}$ & $9.81 \%$ & 0.139 & 1.387 \\
\hline Offs & $1.929^{* * *}$ & $5.16 \%$ & 0.143 & 1.246 \\
\hline Ons $^{2}$ & $-0.132^{* * *}$ & $4.11 \%$ & 0.012 & 1.160 \\
\hline Offs $^{2}$ & $-0.107^{* * *}$ & $2.03 \%$ & 0.014 & 1.093 \\
\hline Lift & $24.01^{* * *}$ & $1.79 \%$ & 1.124 & 1.038 \\
\hline Length & $0.041^{* * *}$ & $1.12 \%$ & 0.002 & 1.023 \\
\hline Avg. Speed & $-0.281^{* * *}$ & $3.90 \%$ & 0.032 & 1.161 \\
\hline Intersection & $2.219^{* * *}$ & $0.47 \%$ & 0.633 & 1.002 \\
\hline Early & $5.700^{* * *}$ & $2.79 \%$ & 0.003 & 1.020 \\
\hline Far-side & $6.493^{* * *}$ & $6.52 \%$ & 0.392 & 1.307 \\
\hline Near-side & $7.252^{* * *}$ & $0.15 \%$ & 0.758 & 1.003 \\
\hline Int-Far & $25.67^{* * *}$ & $2.67 \%$ & 1.185 & 1.053 \\
\hline Int-Near & $-5.260^{* * *}$ & $0.13 \%$ & 1.168 & 1.003 \\
\hline Bus-bay & $0.707^{*}$ & $1.65 \%$ & 0.322 & 1.083 \\
\hline $\mathbf{R}^{2}$ & \multicolumn{4}{|c|}{0.5533} \\
\hline Adj. $R^{2}$ & \multicolumn{4}{|c|}{0.5528} \\
\hline Num. Ob. & \multicolumn{4}{|c|}{15,872} \\
\hline Residual Std. Error & \multicolumn{4}{|c|}{0.4886} \\
\hline
\end{tabular}


Pooled Results

\begin{tabular}{|c|c|c|c|c|}
\hline & \multicolumn{4}{|c|}{ Model (F) - Interval } \\
\hline & Coefficient & $\begin{array}{l}\text { Relative } \\
\text { Contribution }\end{array}$ & Std. Error & $\begin{array}{l}\text { Variance } \\
\text { Inflation } \\
\text { Factor }\end{array}$ \\
\hline Intercept & -0.501 & & 0.788 & \\
\hline Stops & $16.43^{* * *}$ & $13.04 \%$ & 0.392 & 1.450 \\
\hline Ons & $4.041^{* * *}$ & $9.81 \%$ & 0.139 & 1.387 \\
\hline$\overline{O f f s}$ & $1.929^{* * *}$ & $5.16 \%$ & 0.143 & 1.246 \\
\hline$\overline{O n s^{2}}$ & $-0.132^{* * *}$ & $4.11 \%$ & 0.012 & 1.160 \\
\hline$\overline{\text { Offs }^{2}}$ & $-0.107^{* * *}$ & $2.03 \%$ & 0.014 & 1.093 \\
\hline Lift & $24.01^{* * *}$ & $1.79 \%$ & 1.124 & 1.038 \\
\hline Length & $0.041^{* * *}$ & $1.12 \%$ & 0.002 & 1.023 \\
\hline Avg. Speed & $-0.281^{* * *}$ & $3.90 \%$ & 0.032 & 1.161 \\
\hline Intersection & $2.219^{* * *}$ & $0.47 \%$ & 0.633 & 1.002 \\
\hline Early & $5.700^{* * *}$ & $2.79 \%$ & 0.003 & 1.020 \\
\hline Far-side & $6.493^{* * *}$ & $6.52 \%$ & 0.392 & 1.307 \\
\hline Near-side & $7.252^{* * *}$ & $0.15 \%$ & 0.758 & 1.003 \\
\hline Int-Far & $25.67^{* * *}$ & $2.67 \%$ & 1.185 & 1.053 \\
\hline Int-Near & $-5.260^{* * *}$ & $0.13 \%$ & 1.168 & 1.003 \\
\hline Bus-bay & $0.707^{*}$ & $1.65 \%$ & 0.322 & 1.083 \\
\hline$R^{2}$ & \multicolumn{4}{|c|}{0.5533} \\
\hline$\overline{A d j .} R^{2}$ & \multicolumn{4}{|c|}{0.5528} \\
\hline Num. Ob. & \multicolumn{4}{|c|}{15,872} \\
\hline Residual Std. & \multicolumn{4}{|c|}{0.4886} \\
\hline
\end{tabular}




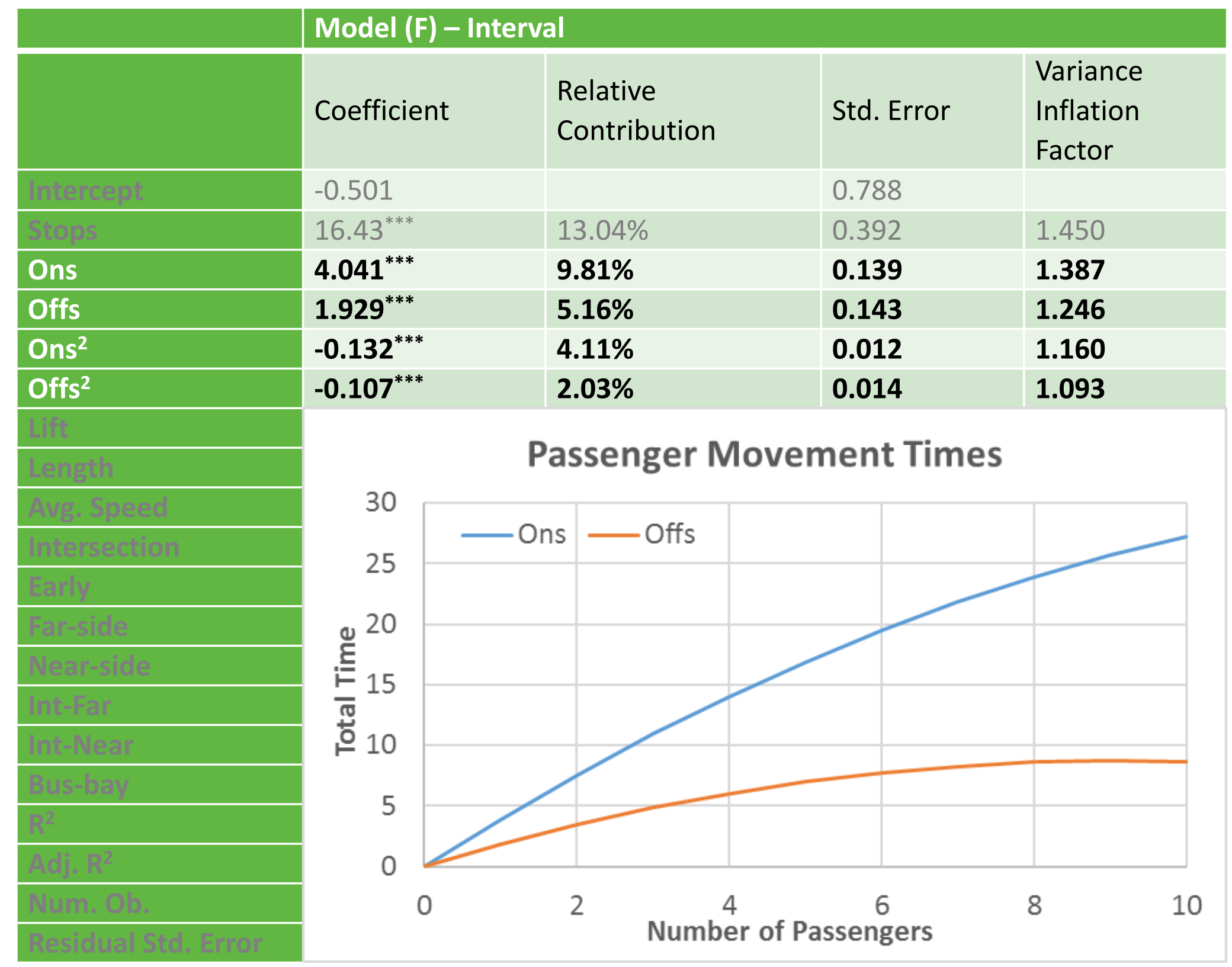


Pooled Results

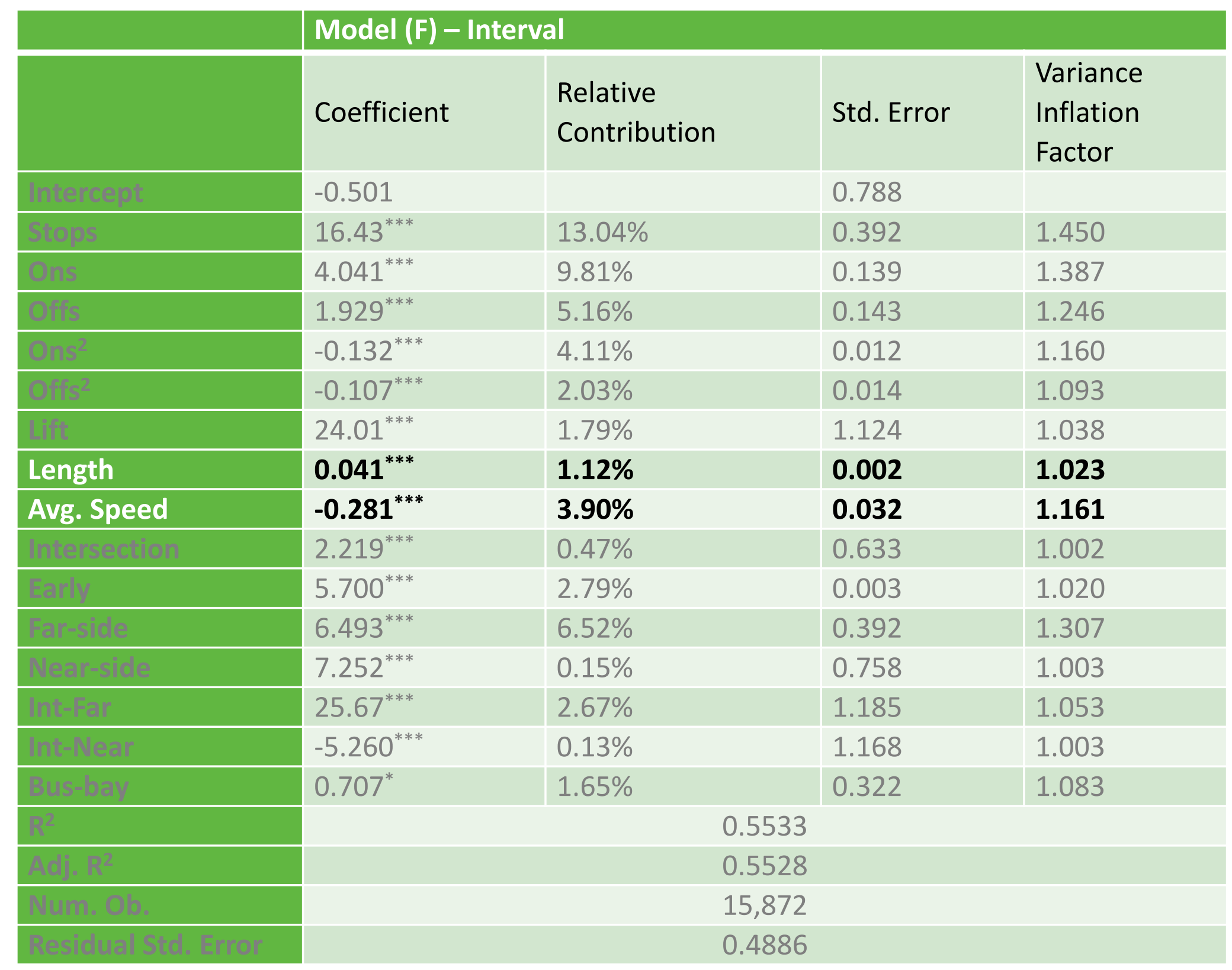


Pooled Results

\begin{tabular}{|c|c|c|c|c|}
\hline & \multicolumn{4}{|c|}{ Model (F) - Interval } \\
\hline & Coefficient & $\begin{array}{l}\text { Relative } \\
\text { Contribution }\end{array}$ & Std. Error & $\begin{array}{l}\text { Variance } \\
\text { Inflation } \\
\text { Factor }\end{array}$ \\
\hline Intercept & -0.501 & & 0.788 & \\
\hline Stops & $16.43^{* * *}$ & $13.04 \%$ & 0.392 & 1.450 \\
\hline Ons & $4.041^{* * *}$ & $9.81 \%$ & 0.139 & 1.387 \\
\hline$\overline{O f f s}$ & $1.929^{* * *}$ & $5.16 \%$ & 0.143 & 1.246 \\
\hline $\mathrm{Ons}^{2}$ & $-0.132^{* * *}$ & $4.11 \%$ & 0.012 & 1.160 \\
\hline Offs $^{2}$ & $-0.107^{* * *}$ & $2.03 \%$ & 0.014 & 1.093 \\
\hline Lift & $24.01^{* * *}$ & $1.79 \%$ & 1.124 & 1.038 \\
\hline Length & $0.041^{* * *}$ & $1.12 \%$ & 0.002 & 1.023 \\
\hline Avg. Speed & $-0.281^{* * *}$ & $3.90 \%$ & 0.032 & 1.161 \\
\hline Intersection & $2.219^{* * *}$ & $0.47 \%$ & 0.633 & 1.002 \\
\hline Early & $5.700^{* * *}$ & $2.79 \%$ & 0.003 & 1.020 \\
\hline Far-side & $6.493^{* * *}$ & $6.52 \%$ & 0.392 & 1.307 \\
\hline Near-side & $7.252^{* * *}$ & $0.15 \%$ & 0.758 & 1.003 \\
\hline Int-Far & $25.67^{* * *}$ & $2.67 \%$ & 1.185 & 1.053 \\
\hline Int-Near & $-5.260^{* * *}$ & $0.13 \%$ & 1.168 & 1.003 \\
\hline Bus-bay & $0.707^{*}$ & $1.65 \%$ & 0.322 & 1.083 \\
\hline $\mathrm{R}^{2}$ & \multicolumn{4}{|c|}{0.5533} \\
\hline Adj. $R^{2}$ & \multicolumn{4}{|c|}{0.5528} \\
\hline Num. Ob. & \multicolumn{4}{|c|}{15,872} \\
\hline Residual Std. & \multicolumn{4}{|c|}{0.4886} \\
\hline
\end{tabular}


Pooled Results

\begin{tabular}{|c|c|c|c|c|}
\hline & \multicolumn{4}{|c|}{ Model (F) - Interval } \\
\hline & Coefficient & $\begin{array}{l}\text { Relative } \\
\text { Contribution }\end{array}$ & Std. Error & $\begin{array}{l}\text { Variance } \\
\text { Inflation } \\
\text { Factor }\end{array}$ \\
\hline Intercept & -0.501 & & 0.788 & \\
\hline Stops & $16.43^{* * *}$ & $13.04 \%$ & 0.392 & 1.450 \\
\hline Ons & $4.041^{* * *}$ & $9.81 \%$ & 0.139 & 1.387 \\
\hline Offs & $1.929^{* * *}$ & $5.16 \%$ & 0.143 & 1.246 \\
\hline Ons $^{2}$ & $-0.132^{* * *}$ & $4.11 \%$ & 0.012 & 1.160 \\
\hline Offs $^{2}$ & $-0.107^{* * *}$ & $2.03 \%$ & 0.014 & 1.093 \\
\hline Lift & $24.01^{* * *}$ & $1.79 \%$ & 1.124 & 1.038 \\
\hline Length & $0.041^{* * *}$ & $1.12 \%$ & 0.002 & 1.023 \\
\hline Avg. Speed & $-0.281^{* * *}$ & $3.90 \%$ & 0.032 & 1.161 \\
\hline Intersection & $2.219^{* * *}$ & $0.47 \%$ & 0.633 & 1.002 \\
\hline Early & $5.700^{* * *}$ & $2.79 \%$ & 0.003 & 1.020 \\
\hline Far-side & $6.493^{* * *}$ & $6.52 \%$ & 0.392 & 1.307 \\
\hline Near-side & $7.252^{* * *}$ & $0.15 \%$ & 0.758 & 1.003 \\
\hline Int-Far & $25.67^{* * *}$ & $2.67 \%$ & 1.185 & 1.053 \\
\hline Int-Near & $-5.260^{* * *}$ & $0.13 \%$ & 1.168 & 1.003 \\
\hline Bus-bay & $0.707^{*}$ & $1.65 \%$ & 0.322 & 1.083 \\
\hline $\mathbf{R}^{2}$ & \multicolumn{4}{|c|}{0.5533} \\
\hline Adj. $R^{2}$ & \multicolumn{4}{|c|}{0.5528} \\
\hline Num. Ob. & \multicolumn{4}{|c|}{15,872} \\
\hline Residual Std. Error & \multicolumn{4}{|c|}{0.4886} \\
\hline
\end{tabular}




\section{Pooled Results}

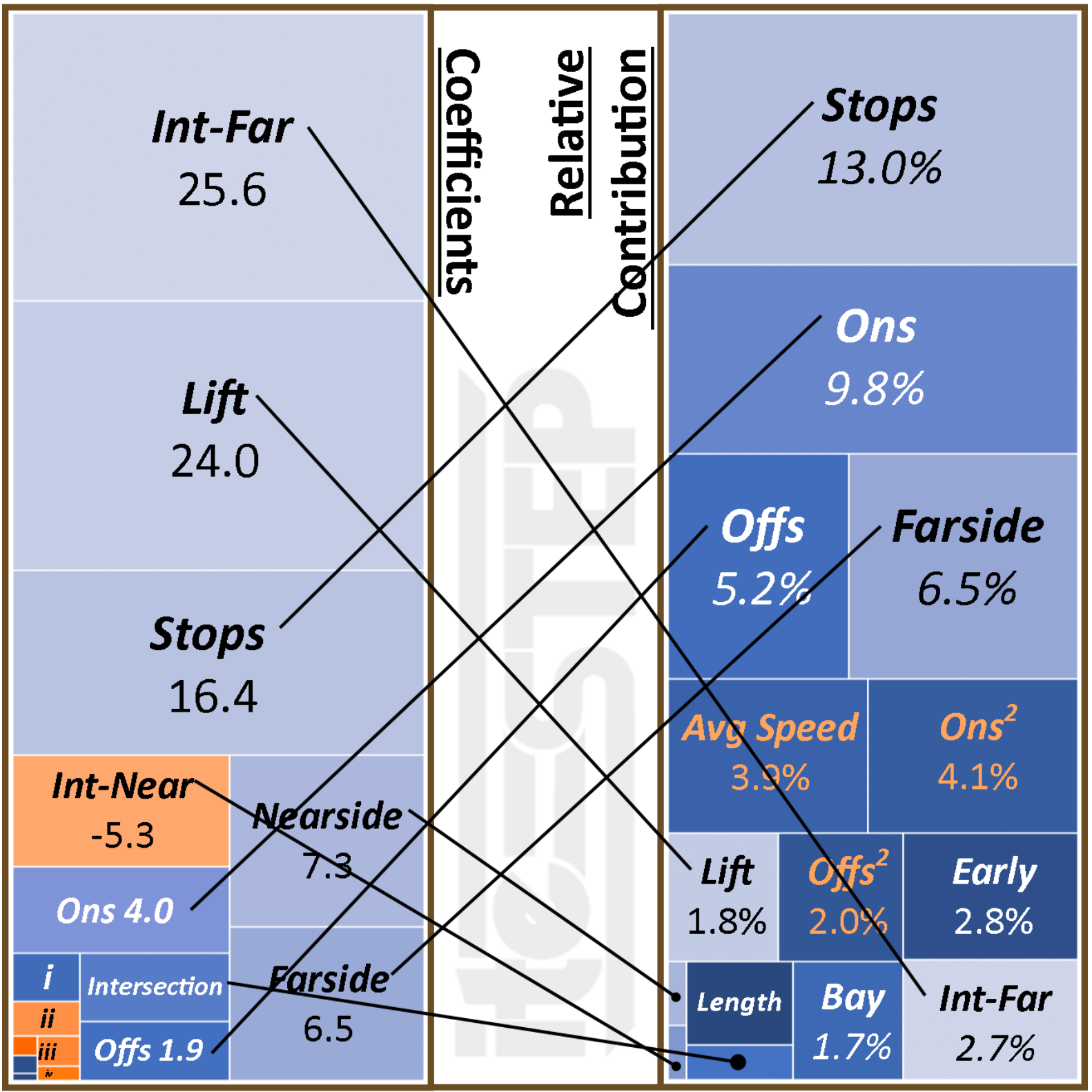
i. Intercept
ii. Avg. Speed
iii. $\mathrm{Ons}^{2}$
iv. Offs $^{2}$
v. Early
vi. Length 


\section{Previous Model Biases}

- Overestimate the coefficients of passenger movements

- Report results that exclude dwells with lift events or near intersections

- Pooled Results:

- Ons, Ons ${ }^{2}$, Offs, Offs ${ }^{2}$, On-time performance, and time-of-day

- $\mathrm{R}^{2}=0.34$

- With lift, $R^{2}=0.28$ 


\section{Conclusions}

- Our Pooled Results account for $55 \%$ of the data variability when looked at all stop types.

- Account for non-linearity in passenger movements, including lift events

- Capture the effect of traffic around a stop

- Capture the effect of traffic signals without having direct data

- More detail in paper 


\section{Thank you! \\ Any Questions?}

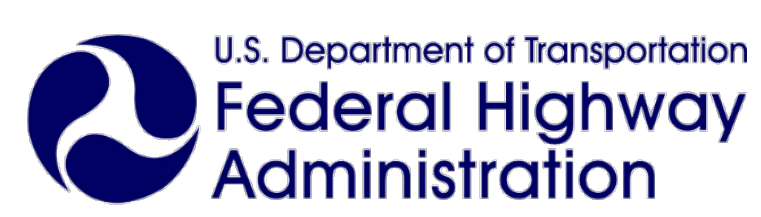

TID Lab TRI (9) MET 